\title{
Philosophiques
}

\section{Commentaire - À quoi servent les faits normatifs ?}

\section{Jocelyne Couture}

Volume 24, numéro 2, automne 1997

URI : https://id.erudit.org/iderudit/027454ar

DOI : https://doi.org/10.7202/027454ar

Aller au sommaire du numéro

Éditeur(s)

Société de philosophie du Québec

ISSN

0316-2923 (imprimé)

1492-1391 (numérique)

Découvrir la revue

Citer cet article

Couture, J. (1997). Commentaire - À quoi servent les faits normatifs ?

Philosophiques, 24(2), 329-335. https://doi.org/10.7202/027454ar d'utilisation que vous pouvez consulter en ligne.

https://apropos.erudit.org/fr/usagers/politique-dutilisation/ 


\title{
COMMENTAIRES
}

\section{À QUOI SERVENT IES FAITS NORMAIIFS ?}

\author{
PAR \\ Jocelyne COUture
}

Selon Charles Larmore, la raison pour laquelle plusieurs philosophes rejettent le réalisme moral - qu'il défend pour sa part - est qu'ils répugnent à admettre l'existence, indépendante de la connaissance que nous pourrions en avoir, de faits moraux et, plus généralement, de faits normatifs. Je ne sais pas qui sont les philosophes auxquels pense Larmore, mais il me semble plutôt que pour plusieurs philosophes contemporains, du moins pour ceux qui se sentent à l'aise à l'intérieur de la tradition quinienne, les questions d'ontologie se résolvent sur la base de considérations pragmatiques. C'est un point de vue que je partage et qui, à la suite de la belle conférence que vient de prononcer le professeur Larmore, m'incite à répliquer par une question : à quoi servent les faits normatifs ? Qu'est-ce qu'ils permettent de comprendre, ou de mieux comprendre, au sujet de nos raisons d'agir et de croire, au sujet de nos croyances normatives et de leur source et au sujet de la morale, du raisonnement moral et de nos croyances morales? On pourrait donner à cette question beaucoup d'ampleur. Je me contenterai ici de la soulever à propos de trois des thèmes qu'a abordés Charles Larmore dans sa conférence : celui du rôle de la philosophie morale, celui de l'autonomie de la morale et, finalement, celui de la connaissance normative.

Je commencerai par une remarque générale concernant le sort réservé à la philosophie morale dans l'approche proposée par Larmore. L'une des choses qui m'a souvent frappée dans ses plus récents écrits, et qui a peut-être frappé aussi les philosophes qui viennent d'entendre sa conférence, est que sa critique de la philosophie morale - de la théorie morale - prend appui sur une conception du raisonnement de l'agent moral en contexte de décision. C'est le point de départ de la conférence que nous venons d'entendre. "Tout homme réfléchi », dit Larmore, a s'est au moins une fois dans sa vie demandé "Pourquoi faut-il être juste?" ". Et lorsqu'il se pose cette question, poursuit Larmore, il le fait parce qu'il a déjà des convictions morales et que celles-ci entrent en conflit avec ses intérêts d'une autre nature. Les arguments qui ont suivi suggèrent certainement que le philosophe moral, lorsqu'il cherche à justifier ou à asseoir des principes moraux, devrait s'en tenir aux manières honnêtes de l'homme réfléchi. C'est aussi ce que Charles Larmore semble vouloir confirmer lorsqu'il suggère que 
si elle reconnaissait enfin l'autonomie de la morale, la philosophie morale pourrait bien ne plus avoir grand-chose à faire. Je pense que Larmore a raison de soupçonner qu'en conséquence des vues qu'il a développées ici, la philosophie morale ne se distinguerait plus guère d'une morale de l'homme réfléchi et que la première ferait mieux, somme toute, de céder le pas à la seconde. En fait, je me propose d'ajouter à ce qui n'était, dans sa conférence, qu'une simple conjecture en soutenant que la disparition appréhendée de la philosophie morale est une conséquence implacable de ses vues. Mais contrairement à Larmore, je ne crois pas que ce soit une conséquence de l'autonomie de la morale. Je pense plutôt qu'il s'agit d'une conséquence, peu réjouissante, du réalisme normatif que Larmore tient à défendre. Je ferai d'abord quelques observations au sujet de ce qu'implique -- et de ce que n'implique pas - pour la philosophie morale, une thèse comme celle de l'autonomie de la morale.

Les critiques que Larmore adresse aux conceptions de la morale rationnelle insistent sur le fait que celles-ci se basent, pour l'articulation et la justification des jugements moraux, sur des idéaux et des normes qui n'appartiennent pas à la morale. Il en conclut que les auteurs de ces conceptions philosophiques n'ont pas compris, ou voulu comprendre, « que la moralité parle pour elle-même $n$, que "la conscience ne peut chercher que dans elle-même l'autorité de la loi morale " et que de a tout point de vue situé en dehors de la morale, nous ne pouvons bien comprendre ni les exigences de la morale ni les attitudes qu'il nous faut avoir à leur égard ". Ainsi formulées, les contraintes assorties à l'autonomie de la morale porteraient sur les processus par lesquels nous pouvons parvenir à identifier les contenus de la morale. Le reproche que Larmore adresse aux philosophes moraux qu'il prend à parti est donc d'avoir voulu se soustraire aux exigences d'un tel processus.

Dans cette critique des conceptions de la morale rationnelle, Larmore est apparemment insensible au fait que si le philosophe et l'homme réfléchi affichent des façons différentes de raisonner lorsqu'il est question de morale, cela tient habituellement au fait, premièrement, que le contexte de leur réflexion est différent et, deuxièmement, qu'ils ont en tête des questions différentes. Ce qui devrait entrainer, il me semble, que ce que le philosophe dit des sources rationnelles de la morale ne doit pas, sauf indications contraires, être tenu pour une description, ni des raisonnements qui l'ont luimême amené à ses conclusions morales, ni du raisonnement de l'homme réfléchi en situation de décision. N'étant pas une description de cela, ces conceptions philosophiques ne nient ni n'affirment quoi que ce soit au sujet du fonctionnement de la moralité dans ces contextes. Lorsque Hobbes nous dit comment la moralité doit être conçue pour les fins de la philosophie politique, il ne dit rien de la façon dont l'homme réfléchi - lui même, Hobbes, étant inclus - raisonne lorsqu'il doit prendre une décision en matière de morale. Il ne dit rien non plus de ce qui a pu l'amener lui-même à vouloir formuler des principes moraux. Et a fortiori il n'y a rien dans sa théorie qui contredise la thèse selon laquelle nous raisonnons dans des contextes habituels de décision, à partir de nos convictions morales. La même chose pourrait être dite de la conception philosophique proposée par Christine Korsgaard ; l'élaboration et la justification philosophique d'une doctrine morale ne sont pas des modélisations du raisonnement moral, tel qu'on voudrait l'imputer à l'agent moral empirique. 
Le philosophe, bref, peut reconnaître l'autonomie de la morale tout en se donnant des objectifs - explicatifs ou justificatifs - limités qui ne l'obligent pas à rendre compte, dans ses théories, de l'autonomie de la morale. De ce point de vue, tout ce que Larmore pourrait reprocher aux conceptions de la morale rationnelle sur la seule base de leurs composantes théoriques, ce n'est pas de nier l'autonomie de la morale, ni même de chercher à se soustraire aux contraintes qui en découlent, mais bien plutôt de ne pas rendre compte de l'autonomie de la morale. Supposons, pour les besoins de la discussion, que tel est le sens de la critique de Larmore : rendre compte de l'autonomie de la morale en montrant comment les principes moraux s'articulent à partir de convictions morales deviendrait, dans cette perspective, un critère de validité pour la philosophie morale. C'est un critère que l'on pourrait vouloir défendre d'un point de vue méta-éthique et qui, dans cette perspective, aurait le mérite de ne pas confondre la théorie morale et la psychologie descriptive de l'agent moral. Un tel critère est amplement satisfait dans une théorie comme celle de John Rawls, par exemple, qui prend son point d'appui sur des convictions et des idéaux moraux dont Rawls soutient qu'ils sont communément partagés. Pourquoi devrions-nous croire que de telles conceptions, parce qu'elles reconnaissent ainsi l'autonomie de la morale, n'ont rien d'autre à nous apprendre que ce que l'homme réfléchi découvre lui-même en raisonnant moralement? On peut très bien partir, et raisonner, de l'intérieur " de nos convictions morales » comme le montre la théorie de Rawls, tout en faisant appel, comme le montre aussi Rawls, à des méthodes d'élucidation et d'élaboration qui ne sont nullement celles de l'agent empirique, et qui contribuent à l'élaboration de normes qui ont valeur de principes moraux. Il me semble que Larmore devrait admettre ici que ce critère - rendre compte de l'autonomie de la morale - est parfaitement compatible avec l'autonomie de la philosophie morale relativement au raisonnement de "l'homme réfléchi ".

Mais il ne peut pas être question d'autonomie pour la philosophie morale lorsqu'on adopte le point de vue du réalisme normatif. Car en vertu du réalisme normatif, tout ce qu'on peut énumérer comme faisant partie des procédés méthodologiques, techniques de preuve et de modélisation usités en philosophie morale, et qui la distinguent des simples raisonnements de l'homme réfléchi, devrait témoigner de l'existence de faits normatifs. Or, et c'est ici que le bât blesse pour une approche comme celle de Larmore, ces faits normatifs ne sont pas des faits moraux, mais plutôt des faits relatifs aux normes intellectuelles, méthodologiques, théoriques qui guident la réflexion du philosophe moral. Dans la perspective du réalisme normatif, la référence à de tels faits va à l'encontre de l'autonomie de la morale qui, comme le soutient Larmore, implique l'homogénéité du domaine des faits auxquels la morale fait appel. Pour se plier aux exigences d'une morale autonome dans le cadre du réalisme normatif, le philosophe moral doit done renoncer à tout ce qui pourrait distinguer ses raisonnements de ceux de l'homme réfléchi. En conséquence de quoi nous devons bien conclure qu'il n'y a rien d'autre en matière de morale que ce que l'homme réfléchi peut lui-même découvrir. Ni la normativité ni les contenus normatifs ne sont, pour Larmore, "quelque chose que la raison construit $\boldsymbol{~}$.

La philosophie morale, même à ses meilleurs moments, n'a pas connu que des heures de gloire. Les philosophes moraux ont souvent affiché une confiance démesurée dans les moyens que leur offrait la philosophie et dans leurs propres capacités de dire l'universel en matière de morale. Dans ces 
excès mêmes, la philosophie morale témoigne du projet et de l'espoir qui l'ont toujours portée, à savoir le projet de réfléchir, de manière patiente, méticuleuse et ordonnée, sur notre propre condition, et l'espoir qu'une telle réflexion nous conduise à une meilleure compréhension de la moralité, de nos comportements moraux et des valeurs qui, étant données nos circonstances, devraient guider nos pratiques individuelles et sociales. Dans ses moments fastes, la philosophie morale a été non seulement le lieu, mais l'instrument d'une telle réflexion. Ce que nous propose Larmore ici, en dépit de son intention déclarée de réhabiliter la connaissance morale et philosophique, c'est de renoncer à la philosophie morale au profit de la morale spontanée de l'homme réfléchi. Et c'est sur la foi d'un argument. ontologique que nous devrions nous y résoudre. Ma position est que si la croyance en des faits normatifs a comme conséquence de nous détourner d'un instrument de réflexion, non point parfait, mais parfois efficace en matière de morale, alors c'est cette croyance qu'il faut abandonner et non pas la philosophie morale.

Ces remarques m'amènent au troisième et dernier thème que je voulais aborder, celui de la connaissance normative. Seul le réalisme normatif, nous dit Larmore, peut nous permettre de réhabiliter la philosophie et la morale dans leur statut de connaissance. S'il n'existe pas de faits authentiquement normatifs, écrit Larmore, " comment peut-on dire des croyances normatives, c'est-à-dire des croyances affirmant qu'il faut croire ceci ou faire cela, qu'elles sont vraies ou fausses? ". Ainsi, les croyances normatives seraient vraies ou fausses, selon Larmore, en vertu de faits normatifs et ceux-ci sont nos raisons de croire ou d'agir. Mais ces raisons, poursuit-il, « ne sont pas les autres croyances que nous avons et qui font partie de la justification d'une certaine proposition ", c'est-à-dire des croyances qui permettraient de déterminer si cette proposition est vraie ou fausse. Larmore a certainement raison, si telle est bien son intention, d'établir une distinction entre les croyances qui permettent de déterminer la vérité d'une proposition et celles qui nous amènent à faire de cette proposition le contenu d'une croyance. Par exemple, ma croyance que la neige est blanche ne serait pas entièrement expliquée en disant que j'ai observé le fait (naturel) de la blancheur de la neige fraîchement tombée, qui rend vraie cette proposition. Pour expliquer cette croyance, encore faut-il faire appel à d'autres croyances, normatives celles-là, comme, par exemple, la croyance selon laquelle ce qui est vrai mérite d'être cru. Or, il faut bien remarquer qu'en dépit de la distinction ontologique entre les faits naturels et les faits normatifs, qui motive apparemment pour Larmore une démarcation entre la vérité d'une proposition et la croyance en cette proposition, c'est bien toujours la véracité des croyances normatives, plutôt que les raisons que l'on pourrait avoir de les adopter, qui en font, dans son approche, des raisons de croire. Si, comme le soutient Larmore, une croyance normative " $\mathrm{x}$ " renvoie à un fait normatif qui constitue une raison de croire " $y$ ", alors mes raisons de croire " $y$ "se réduisent bel et bien aux croyances qui servent à déterminer la vérité de « $\mathrm{x}$ ». Mais si, comme le suggère Larmore, le fait naturel qui rend vraie l'affirmation " la neige est blanche " ne suffit à expliquer la croyance que la neige est blanche, alors le fait normatif qui rend a $x$ "vraie ne devrait pas non plus suffire à expliquer ma croyance en " $x$ ". Il résulte de ceci que ce que Larmore considérerait comme une raison de croire a $y$ * n'explique nullement ma croyance en a $y$ ». Encore faudrait-il montrer qu'il existe des raisons d'adopter la croyance normative « $\mathbf{x}$ *dont la véracité est impliquée dans la 
croyance que " $y$ ». De telles raisons devraient faire intervenir de nouveaux faits normatifs révélant, par exemple, que ce qui est vrai dans le domaine normatif mérite d'être cru. Tout comme dans le cas précédent, ce fait normatif, qui établirait la vérité d'une croyance $\propto \mathrm{z}{ }^{\star}$, constituerait la raison d'adopter la croyance " $x »$. Et un autre fait normatif devrait à son tour expliquer l'adoption de la croyance $\propto \mathrm{z}$ n. Mais où s'arrête cette escalade ? La régression à l'infini que l'on peut imaginer ici semble impliquer qu'une croyance normative concernant ce que l'on doit croire, ou bien ne pourra être expliquée que par la vérité de ce qu'on croit (contrairement à ce que soutient Larmore), ou bien ne pourra jamais être justifiée. C'est bien ce qu'avait découvert Bertrand Russell au sujet du principe d'induction. Il faudrait voir ici comment la relation que Charles Larmore pose entre les raisons et les faits - fussent-ils normatifs - , entre la croyance normative et la vérité, réussit à éviter l'impasse.

La conséquence de ceci pour ce qui est des croyances concernant ce que l'on doit faire, c'est-à-dire pour les croyances à strictement parler morales, est également inadmissible aux termes mêmes de la position défendue par Larmore. La souffrance d'autrui, dit-il, n'est pas une raison d'agir. En tant que telle, en effet, elle ne peut être qu'un fait naturel tout au plus susceptible de rendre vraie la croyance qu'autrui souffre. Mais le fait normatif qui pourrait expliquer - supposons-le - l'adoption de la croyance qu'autrui souffre n'est toujours pas une raison d'agir ; ce ne peut être qu'une raison de croire. Et même si un fait spécifiquement moral révélait, par exemple, que la souffrance mérite d'être soulagée, ce fait ne serait toujours qu'une raison d'adopter une croyance concernant ce qui doit être fait et non pas une raison d'agir conformément au contenu de cette croyance. Tant et aussi longtemps que les raisons sont caractérisées en référence à des faits moraux, c'est-à-dire relativement à la vérité des croyances normatives, on ne peut aboutir, au mieux, qu'à des raisons de croire, plutôt qu'à des raisons d'agir. Et ceci ne signifie pas qu'il fajlle conclure, comme le craint Larmore, à l'inertie de la raison -- la raison n'est inerte ni dans l'adoption ni dans le changement ni dans le refus d'adopter des croyances concernant ce qu'il faut croire ou ce qu'il faut faire. On peut même concéder qu'elle n'est pas inerte non plus lorsqu'il s'agit de décirler d'agir conformément aux croyances normatives. La question est plutôt de savoir dans quelle mesure les faits - fussent-ils normatifs - peuvent avoir, sur la raison, l'influence déterminante que Larmore leur suppose.

Larmore ambitionne de réhabiliter la connaissance philosophique et morale en admettant une ontologie de faits normatifs. Il aurait tout aussi bien pu inclure la connaissance scientifique elle-même dans ce projet de réhabilitation car, en vertu de la conception de la connaissance qui sous-tend son entreprise, nulle croyance, fût-elle à propos des faits naturels, ne peut voir le jour sans la présence de faits normatifs. Mais dans ce modèle unifié de la connaissance, comment expliquer que ce que les faits naturels ne peuvent achever en termes de raisons de croire et d'agir, d'autres faits - normatifs, ceux-là - devraient en être capables? Et comment expliquer l'autonomie de la morale alors que la science n'en aurait aucune? Je voudrais suggérer, pour finir, que le modèle de connaissance qui peut rendre compte des conceptions les plus fructueuses et aussi les plus courantes de la connaissance scientifique et de l'activité de théorisation qui y est liée peut aisément, et à moindre frais ontologiques, rendre compte de la nature et du rôle des croyances normatives concernant ce qu'il faut croire et ce qu'il faut faire. 
Il est assez courant, il me semble, de supposer qu'un agent qui a une raison d'agir possède au moins une représentation du monde au temps $t_{1}$, antérieur à l'action qu'il envisage, et une représentation du monde au temps $t_{2}$, tel qu'il résulterait de l'action en question. Sa décision d'agir ou de ne pas agir pourrait ainsi être expliquée en référence à certaines caractéristiques de ces deux représentations du monde, caractéristiques qui constituent dès lors ses raisons d'agir. Je pense que les raisons de croire ne sont guère différentes dans leur structure. J'ai au temps $t_{1}$ une représentation du monde qui sera modifiée au temps $t_{2}$ par l'adoption d'une nouvelle croyance. Que je l'adopte ou non pourra ainsi être expliqué par certaines caractéristiques de ces deux représentations qui constituent dès lors mes raisons de croire.

Quel rôle jouent les faits dans ce dernicr cas? Le fait qu'il y ait devant moi un objet bleu et ma perception de ce fait peuvent bien dans certains cas m'amener à croire qu'il y a dans mon environnement physique immédiat un objet bleu; mais l'irruption soudaine d'une licorne dans mon bureau et ma vision de cette licorne ne m'amèneront jamais à croire qu'il y a une licorne dans mon environnement physique. Les croyances que j'ai déjà concernant le monde m'inviteront plutôt à croire que j'ai été victime d'une hallucination ou que quelqu'un s'est amusé à attacher une corne à la tête de son chien. Ainsi en va-t-il, a fortiori, des croyances plus abstraites que nous entretenons; nos raisons de les entretenir et de les adopter ne résident pas dans d'hypothétiques " faits naturels ", mais dans le réseau des croyances qui, au cours des âges, s'est montré le plus cohérent et, par conséquent, le plus commode. Cette perspective devrait être entièrement compatible avec celle de Larmore puisqu'il admet que la vérité d'une proposition joue un rôle minimal dans l'adoption de la croyance correspondante.

Mais si les faits naturels ont une importance si limitée dans la formation et le maintien de nos croyances, pourquoi devrions-nous penser que d'autres faits - normatifs, ceux-là - peuvent prendre le relais? Que peut-on attendre des faits, normatifs ou autres, et avons-nous conceptuellement besoin de faits normatifs pour expliquer que nous avons des raisons de croire et des raisons d'agir? J'avancerais plutôt que nos croyances nous suffisent. Certaines d'entre elles sont des croyances normatives de part en part, comme celles qui nous amènent à rechercher la cohérence ou l'adéquation de nos croyances, qu'elles soient " naturelles ", morales ou plus généralement normatives; d'autres sont indirectement normatives, en ceci que leur normativité résulte du fait qu'elles soient partie du réseau des croyances déjà admises et qui, à l'usage, se sont révélées fiables. Tel est le cas de la plupart. des croyances qui concernent les normes morales et épistémologiques, mais aussi de ces croyances qui forment ce qu'on appelle la connaissance scientifique. C'est sur la base de toutes ces croyances que nous décidons ou non d'en accepter de nouvelles; ce sont elles qui constituent nos raisons de croire. Et c'est sur la base de ces croyances concernant l'état du monde et les résultats possibles de nos actions que nous décidons ou non d'agir.

Cette façon de concevoir la connaissance et la source de nos raisons ne plairait pas à Charles Larmore puisqu'on y rencontre bien peu les faits et la vérité et puisque, en vertu de cette conception, nul domaine de la connaissance ne peut se prévaloir d'une autonomie par rapport aux autres. Mais c'est une conception qui permet d'expliquer de façon uniforme la connaissance dite empirique et la connaissance dite normative et qui permet de comprendre le rôle de la normativité dans la formation des croyances normatives tout autant que dans la formation des croyances naturelles. 
Contrairement au modèle proposé par Larmore, celui-ci permet de rendre compte de la modification des croyances et de l'adoption de croyances nouvelles. Bien que Larmore attache une grande importance à la réflexion dans la découverte des faits normatifs, on voit mal, en effet, comment la connaissance normative comme il la conçoit pourrait encore laisser place à quelque opération intellectuelle ou rationnelle reliée à l'évaluation critique des croyances que nous entretenons. Les faits, contrairement aux représentations qu'on s'en fait, sont immuables et ils parlent d'eux-mêmes.

Il y a done, il me semble, quelques raisons pragmatiques qui militent contre l'admission d'une ontologie de faits normatifs. La première raison que j'ai avancée est que le réalisme normatif conduit tout droit à l'abandon de la philosophie morale, c'est-à-dire à l'abandon d'une réflexion critique, parfois efficace et souvent éclairante, concernant nos pratiques et nos croyances morales. La deuxième raison est qu'une telle ontologie, non seulement n'est pas requise lorsqu'il s'agit d'expliquer les croyances normatives et le rôle qu'elles jouent dans la constitution des croyances naturelles, mais est de plus incapable de contribuer à donner une explication satisfaisante des unes et des autres. Une troisième raison est que le modèle de connaissance qu'induisent les faits normatifs entre en conflit avec les conceptions les plus fructueuses du rôle et de la fonction des théories scientifiques. Bref, les faits normatifs ne font par partie de nos meilleures explications de la connaissance, des croyances et de l'activité de théorisation.

Il y a aussi une autre raison, dont je n'ai pas parlé jusqu'ici, mais que je voudrais simplement mentionner pour finir. Et cette dernière raison réside dans la redondance explicative que les faits moraux introduisent, à ce qu'il me semble, dans la conception de la morale proposée par Charles Larmore. La thèse de l'autonomie de la morale, par exemple, quoique éminemment contestable, comme je l'ai souligné, est parfaitement claire, comme je l'ai aussi souligné, sans que l'on ait besoin de faire intervenir les faits moraux. De plus, Larmore dispose déjà d'une version non réaliste de l'autonomie de la morale puisqu'il nous dit, par exemple, que nous raisonnons à partir de notre expérience, que " notre sensibilité morale prend forme au moyen de processus de socialisation "et reflète ainsi des préoccupations particulières à notre culture. Pourquoi avons-nous besoin en plus de faits moraux ? Il me semble qu'ils ne contribuent aucumement à faire de l'autonomie de la morale une notion intelligible. Il en va de même, à ce qu'il me semble, pour l'idée de connaissance morale. Les passages que je viens de citer suffiraient amplement à nous dispenser de ces entités surnuméraires que sont les faits normatifs.

Département de philosophie

Université du Québec à Montréal 